\title{
BIÓPSIA PULMONAR PERCUTÂNEA GUIADA POR TOMOGRAFIA COMPUTADORIZADA: DADOS DE UM HOSPITAL*
}

\author{
Emílio Humberto Carazzai ${ }^{1}$, Maristela Andreosi ${ }^{2}$, Fábio Mota Gonzalez ${ }^{3}$, Sandra de Quadros \\ Uzêda Gonzalez ${ }^{4}$, Olger de Souza Tornin ${ }^{5}$, Marcelo D'Andrea Rossi ${ }^{6}$
}

Resumo OBJETIVO: Apresentar a experiência do serviço de radiologia do Hospital Santa Cecília, São Paulo, SP, no manejo das biópsias pulmonares por aspiração através de agulha fina e biópsias por fragmentos guiadas por tomografia computadorizada e a análise de sua importância e associação com suas complicações. MATERIAIS E MÉTODOS: Foram analisadas 168 biópsias guiadas por tomografia, sendo 84 em homens e 84 em mulheres. Utilizou-se a técnica de biópsia por aspiração por agulha fina em 64 pacientes, a técnica de biópsia por fragmento em 68 pacientes e ambas as técnicas em 36 pacientes. RESULTADOS: Pneumotórax ocorreu em 38 pacientes e a hemorragia pulmonar, em dez pacientes. As dimensões das lesões biopsiadas variaram de $0,5 \mathrm{~cm}$ até $15 \mathrm{~cm}$. $O$ diagnóstico foi realizado na primeira tentativa em 132 casos e na segunda tentativa em dez casos. CONCLUSÃO: A acurácia das biópsias aspirativas por agulha fina e por fragmento de lesões pulmonares depende do tamanho da lesão e da colaboração do paciente. Essas técnicas são relativamente seguras e têm acurácia diagnóstica elevada quando feitas por um profissional experiente.

Unitermos: Biópsia; Câncer; Tomografia computadorizada.

Abstract Percutaneous computed tomography-guided biopsy of the lung: data from a hospital.

OBJECTIVE: To present the experience of Santa Cecília Hospital (São Paulo, SP, Brazil) radiology service in the handling of computed tomography-guided fine-needle aspiration biopsy and percutaneous core biopsy of pulmonary lesions, analyzing their importance and associated complications. MATERIALS AND METHODS: One hundred and sixty-eight computed tomography-guided biopsies were performed in 84 men and 84 women. Sixty-four patients underwent fine-needle aspiration biopsy, 68 underwent percutaneous core biopsy and 36 patients underwent both techniques. RESULTS: Pneumothorax occurred in $\mathbf{3 8}$ patients, and pulmonary hemorrhage in then cases. The biopsied lesions ranged in size from 0.5 to $15 \mathrm{~cm}$. The diagnosis was achieved at the first attempt in 132 cases and at the second attempt in ten cases. CONCLUSION: The accuracy of fine-needle aspiration and percutaneous core biopsies depends both on the size of the lesion and the patient's cooperation. These techniques are relatively safe and present a high diagnostic accuracy when performed by an experienced professional.

Keywords: Biopsy; Cancer; Computed tomography.

\section{INTRODUÇÃO}

A realização de biópsias pulmonares guiadas por tomografia computadorizada (TC), seja por aspiração ou por retirada de fragmentos do tecido, é procedimento rotineiramente executado em países europeus, nos Estados Unidos da América e no

* Trabalho realizado no Hospital Santa Cecília, São Paulo, SP 1. Médico Radiologista da Maximagem, Hospital São Luís e Hospital Santa Cecília.

2. Médica Radiologista, Assistente do Setor de Ultra-sonografia da Medimagem - Hospital Beneficência Portuguesa de São Paulo.

3. Mestre em Ciências da Saúde pelo Hospital Heliópolis.

4. Mestre em Morfologia pela Universidade Federal de São Paulo-Escola Paulista de Medicina.

5. Mestrando em Ciências da Saúde pelo Hospital Heliópolis, Prática Profissionalizante em Ressonância Magnética pela Universidade de São Paulo.

6. Diretor Clínico da Maximagem, Médico Radiologista do Hospital São Luís.

Correspondência: Dr. Olger de Souza Tornin. Rua Oscar Freire, 1811, ap. 106, Pinheiros. São Paulo, SP, 05409-011. E-mail: olger1@uol.com.br

Recebido para publicação em 2/9/2005. Aceito, após revisão, em 16/12/2005
Japão, com comprovada especificidade, acurácia e sensibilidade no diagnóstico de lesões pulmonares ${ }^{(\mathbf{1}-\mathbf{5})}$.

As principais complicações relacionadas a esses métodos são o pneumotórax e a hemorragia pulmonar perilesional, respectivamente. A frequiência de pneumotórax citada na literatura, após esses tipos de procedimentos, variou de $11,5 \%$ a $62 \%{ }^{(\mathbf{1 - 1 2})}$, sendo a maioria de pequeno volume, não necessitando de tratamento específico.

No Brasil, existem poucos trabalhos publicados relatando o uso de biópsia percutânea guiada por TC visando ao diagnóstico de afecções pulmonares ${ }^{(\mathbf{4 , 1 3 , 1 4})}$. Além disso, apesar da ampla aceitação e importância deste método na literatura mundial e de sua facilidade de execução após treinamento, é relativamente pouco executado em nosso país, de uma forma geral, permanecendo como procedimento de rotina, apenas em um número restrito de instituições.
O objetivo deste estudo é orientar os profissionais a respeito das técnicas e principais complicações das biópsias pulmonares guiadas por TC, além de apresentar dados epidemiológicos, algo escasso na literatura nacional.

\section{MATERIAIS E MÉTODOS}

Foram analisados os resultado de 168 biópsias torácicas guiadas por TC realizadas no Hospital Santa Cecília, localizado na cidade de São Paulo, no período de setembro de 1997 a abril de 2003.

O grupo de pacientes era composto por 84 homens e 84 mulheres. A idade dos pacientes variou entre 14 e 81 anos, com média de 57,9 anos.

Utilizamos a técnica de biópsia aspirativa por agulha fina em 64 pacientes, a técnica de biópsia por fragmento em 68 pacientes e ambas as técnicas em 36 pacientes. 
Todas as biópsias foram guiadas por TC nos seguintes aparelhos: Toshiba X-speed, CT scanner e Toshiba Medical System.

Antes da realização da biópsia, o radiologista orientava o paciente a respeito do procedimento, buscando tranquiilizá-lo. As possíveis complicações eram explicadas quando da obtenção do consentimento informado.

No momento prévio ao exame, era realizado um treinamento de controle rítmico da respiração, orientando o paciente para não inspirar profundamente durante o procedimento, mas com volume inspiratório o mais constante possível, buscando, dessa forma, reduzir o estresse sobre o local da pleura que iria ser perfurado. Eram feitas algumas imagens sobre a lesão para certificar-se da eficácia dos movimentos respiratórios realizados pelo paciente.

Posicionava-se, então, o marcador cutâneo no local onde havia sido obtido o trajeto mais curto entre a pele e a lesão, almejando não atingir, durante o trajeto, estruturas importantes.

Posteriormente, era realizada uma imagem sobre o marcador para confirmar a relação deste com a lesão.

Retirava-se o marcador quando o paciente já fora assinalado com tinta. A seguir, realizava-se assepsia com iodo ou com álcool iodado e colocavam-se os campos estéreis, e injetava-se anestesia local com xilocaína a $2 \%$ (cerca de $8 \mathrm{ml}$ ). Como guia, deixa-se a própria agulha anestésica no local da punção, para eventualmente mudar a posição ou a angulação do trajeto da biópsia.

Em relação à escolha da técnica, optouse pela realização de biópsia por fragmentos nas lesões em contato com a pleura e biópsia por aspiração por agulha fina nas lesões mais distantes, visando a reduzir a freqüência de pneumotórax. Nas lesões maiores utilizou-se a técnica combinada, desde que estivessem em contato com a pleura.

As agulhas utilizadas foram: Gallini SRL "automatic guillotine needle" (de 16 e 18 gauge) e Shiba "citological aspirating needle" (de 22 gauge), ambas com $10 \mathrm{~cm}$ ou $15 \mathrm{~cm}$.

Realizava-se a introdução da agulha procurando não transgredir a pleura neste momento. Então era realizada uma nova imagem para certificar a direção e aferir a profundidade da lesão e, a seguir, introduzia-se a agulha até o ponto desejado, para realizar novo controle tomográfico.

Confirmando-se a posição no interior da lesão, retirava-se o fragmento, o qual era colocado, de imediato, em recipiente apropriado para esse fim ou aspirava-se o material e realizava-se o esfregaço em lâminas, encaminhando, posteriormente, à anatomia patológica.

É necessário serem feitas imagens de controle durante e após o término, colocando-se o paciente deitado sobre o local da punção.

Em caso de pneumotórax, adota-se a conduta mostrada no Quadro 1.

\section{RESULTADOS}

A média de tempo de realização dos exames analisados foi de 19 minutos, sendo o exame mais rápido em 10 minutos e o mais demorado em 46 minutos. O tempo considerado foi o tempo transcorrido entre a primeira imagem para localização do marcador cutâneo até a imagem de controle após o procedimento.

As biópsias aspirativas constituíram $38,1 \%$ (64 casos), as por fragmentos representaram 40,48\% (68 casos) e as combinadas, $21,42 \%$ (36 casos).

As dimensões das lesões variaram entre $0,5 \mathrm{~cm}$ e $15 \mathrm{~cm}$, sendo a média de 4,5 $\mathrm{cm}$. Quarenta lesões mediram 2,0 cm ou menos.

A maioria das lesões localizava-se junto à pleura (134 do total). A distância da pleura até a lesão variou de $0,5 \mathrm{~cm}$ a $5,0 \mathrm{~cm}$, com média de $2,45 \mathrm{~cm}$.

$\mathrm{O}$ maior número de transgressões da pleura em um procedimento foi de seis e o menor foi de um. O número médio de transgressões foi de 1,44 .

O diagnóstico foi realizado na primeira tentativa em 132 casos e na segunda tentativa em dez casos.

O número de biópsias inconclusivas foi de 26 , sendo dessas 20 aspirativas e seis por fragmentos.

Trinta e oito pacientes apresentaram pneumotórax durante o procedimento ou logo após (Figura 4). Desses 38 pacientes, apenas dois deles foram submetidos à as-

\section{Quadro 1.}

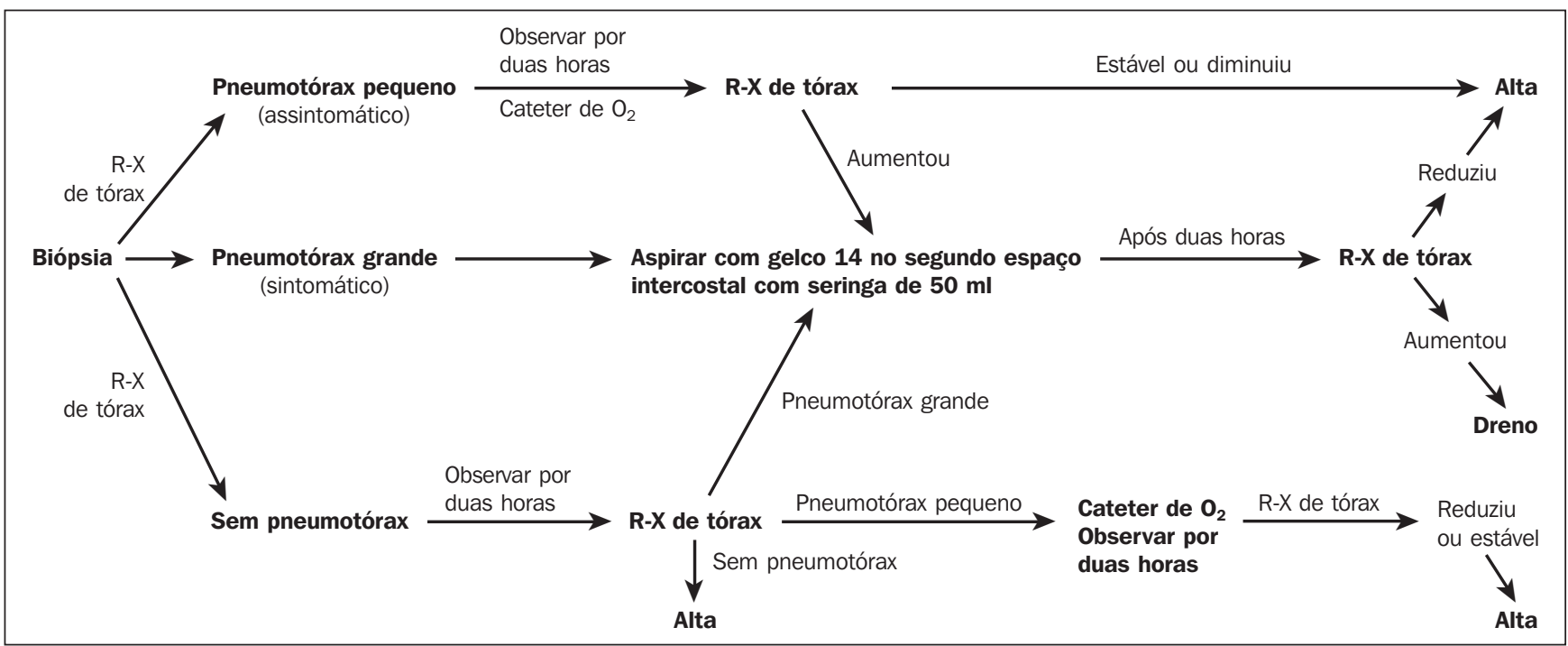


piração com gelco 14 no segundo espaço intercostal na linha hemiclavicular, sem necessidade de colocação de dreno de tórax posteriormente.

Apenas dez pacientes $(5,9 \%)$ apresentaram hemorragia adjacente à lesão, sem maiores complicações (Figura 5). Não houve relatos de hemoptise significativa pós-biópsia.

O número de lesões neoplásicas diagnosticadas no exame anatomopatológico foi de $128(76,15 \%)$, as lesões benignas foram $14(8,33 \%)$ e as biópsias inespecificas/inconclusivas, $26(15,47 \%)$. Isso representa $84,52 \%$ de biópsias diagnósticas. Das lesões com 2,0 cm ou menos (40 lesões), 28 forneceram material suficiente para o diagnóstico. Oito eram benignas e 20 eram malignas.
Os tipos histológicos das neoplasias encontrados foram: adenocarcinoma (24 casos), carcinoma de pequenas células (22 casos), carcinoma de grandes células (20 casos), carcinoma espinocelular (nove casos), carcinoma bronquioloalveolar (três casos), carcinoma neuroendócrino (dois casos), carcinoma broncogênico (em dois casos não foi possível definir o subtipo), tumor neuroectodérmico primitivo (quatro casos), tumor carcinóide (dez casos), rabdomiossarcoma (quatro casos), schwannoma (seis casos), melanoma (dois casos), metástases (12 casos), linfoma (dois casos), timoma (dois casos), neurofibrossarcoma (dois casos) e sarcoma de Kaposi (dois casos) (Figuras 1 a 4 ).

No grupo de lesões benignas foram encontrados: hamartoma condróide (cinco
Figura 1. Paciente de 50 anos de idade, com diagnóstico de adenocarcinoma pouco diferenciado no qual foi realizada biópsia aspirativa por agulha fina. Marcador cutâneo adjacente à lesão nodular periférica com $2,0 \mathrm{~cm}$ localizada no lobo inferior do pulmão esquerdo. 0 paciente se encontra em decúbito ventral para um melhor acesso à lesão.

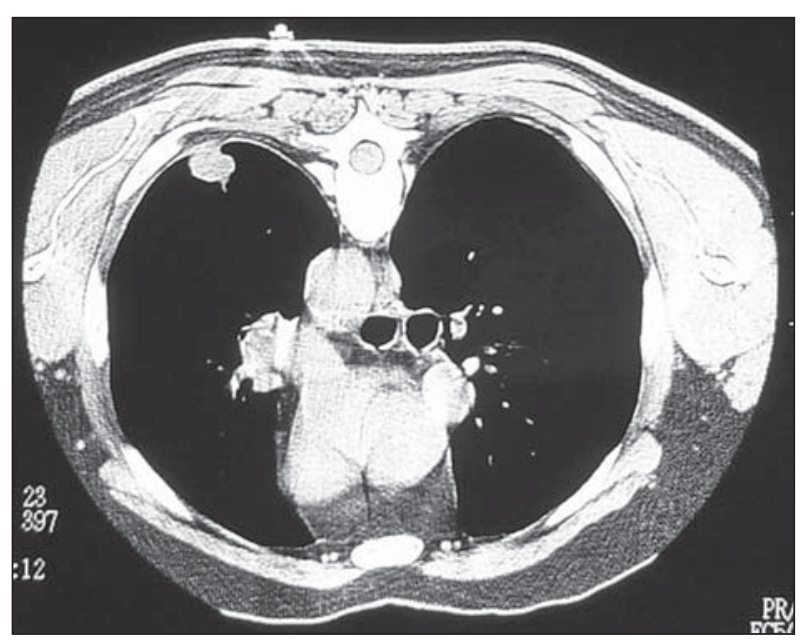

casos), granuloma tuberculoso (cinco casos), sarcoidose (um caso) e abscesso (três casos).

\section{DISCUSSÃO}

Quando detectados nódulos ou massas pulmonares, o principal objetivo é determinar se a lesão é benigna ou maligna. Para essa finalidade, a biópsia percutânea torácica guiada por TC é fundamental.

Nesse estudo, observou-se que o diâmetro da lesão foi fator significante na acurácia diagnóstica. Quanto menor a lesão, menor a acurácia. Alguns estudos já descreveram esses achados, como o de vanSonnenberg et $a l .{ }^{(\mathbf{1})}$, no qual relatou-se acurácia diagnóstica de $90 \%$ para lesões de 3,1 $\mathrm{cm}$ a 4,0 cm de diâmetro, $89,3 \%$ para lesões de $2,1 \mathrm{~cm}$ a $3,0 \mathrm{~cm}, 83,9 \%$ para lesões de $1,1 \mathrm{~cm}$ a $2,0 \mathrm{~cm}$ e $73,9 \%$ para lesões de $0,3 \mathrm{~cm}$ a $1,0 \mathrm{~cm}$. Já para Li et al. ${ }^{(\mathbf{1 2})}$, a acurácia foi de $74 \%$ para lesões menores que 1,5 cm e $96 \%$ para lesões maiores que 1,5 $\mathrm{cm}$. Este último trabalho obteve acurácia geral de $84,5 \%$, não levando em consideração o tamanho da lesão.

Considerando apenas as lesões menores que 2,0 cm, a acurácia diminui para cerca de $70 \%$. Esse índice diminuiria ainda mais se considerássemos apenas as biópsias aspirativas por agulha fina dessas lesões, sem a presença do patologista no momento do procedimento. Ainda em relação à acurácia sem presença do patologista no local, Klein et al. ${ }^{(\mathbf{1 5})}$ relataram acurácia de $88 \%$

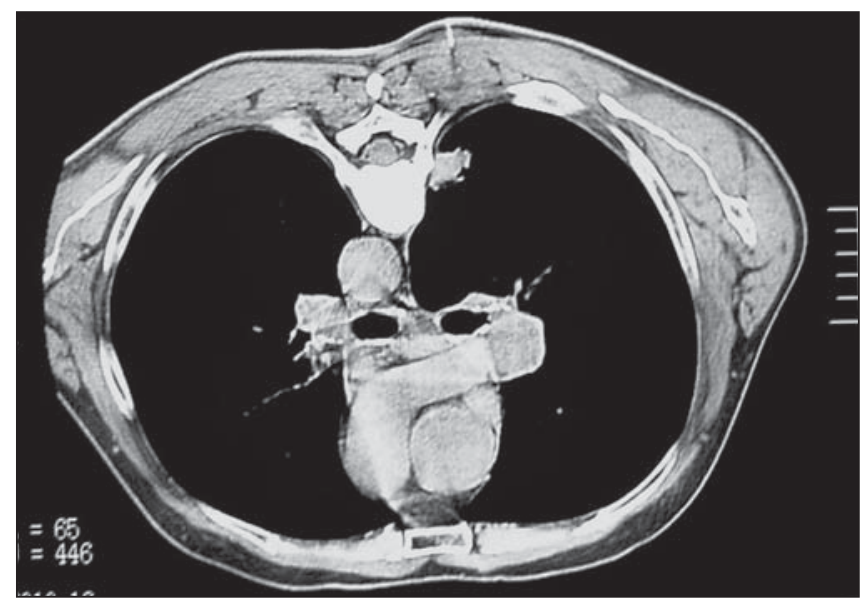

A

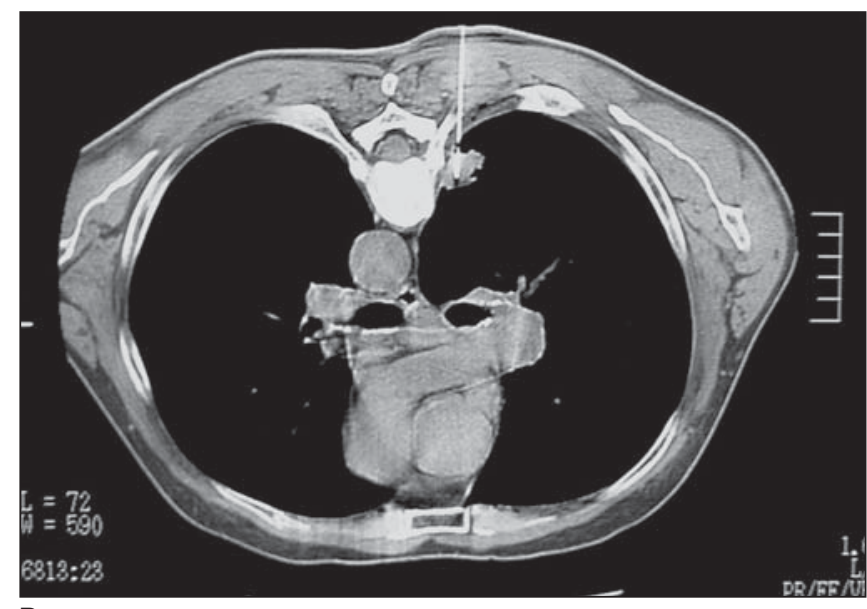

B

Figura 2. Paciente de 68 anos de idade, tabagista, com tumor carcinóide no qual se observa nódulo pulmonar paravertebral à direita e linfonodos hilares ipsilaterais. A: Imagem obtida antes da transfixação da pleura para confirmar o trajeto da agulha. B: Agulha de punção aspirativa localizada no interior da lesão. 


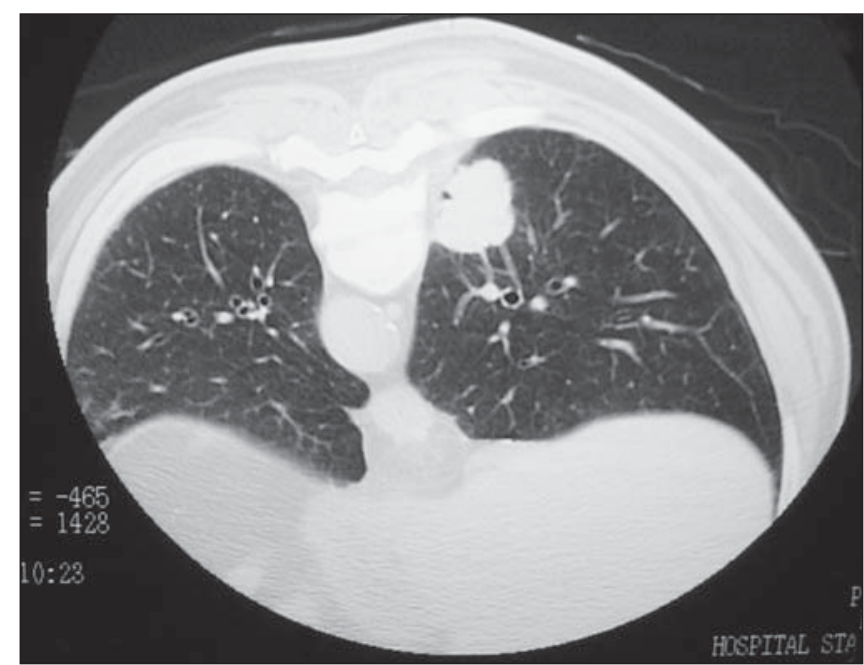

A

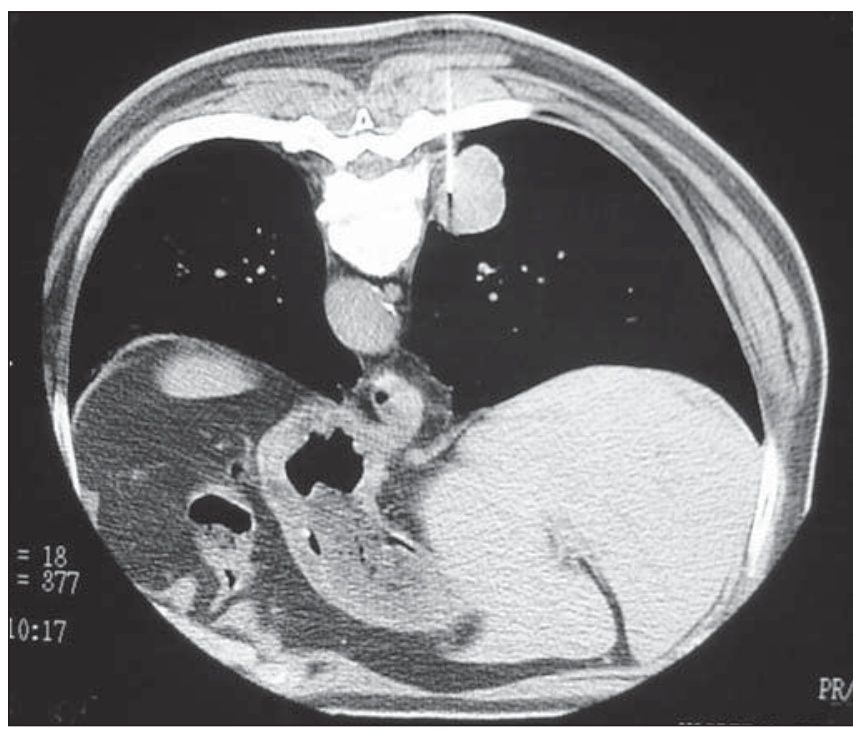

Figura 3. Paciente de 75 anos de idade, com carcinoma espinocelular. A: Massa localizada no lobo inferior do pulmão direito, vista na janela pulmonar, com o paciente em decúbito ventral. B: Agulha de biópsia por fragmento no interior da lesão. Imagem obtida para confirmação da posição da agulha.

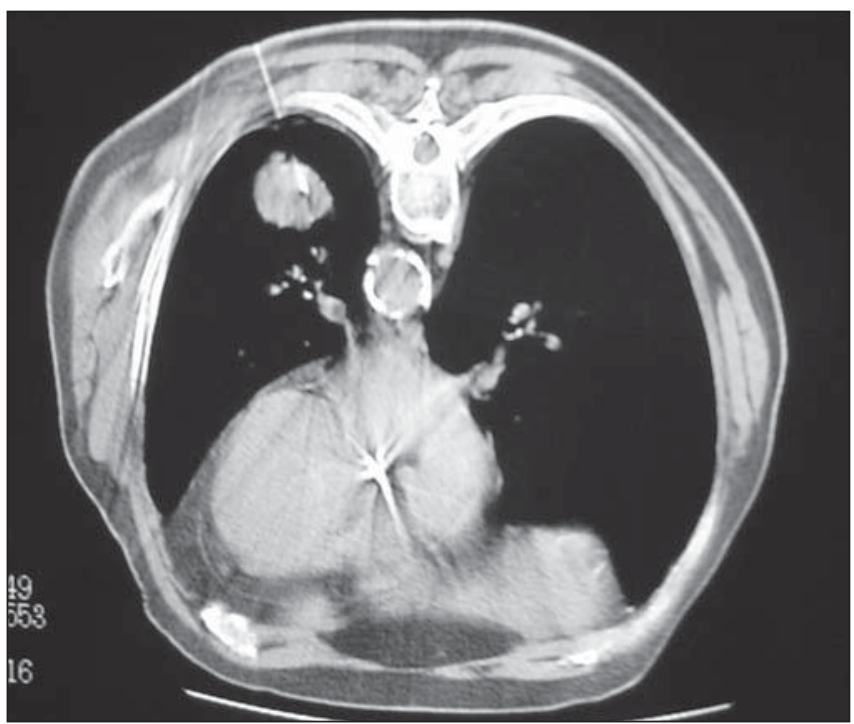

A

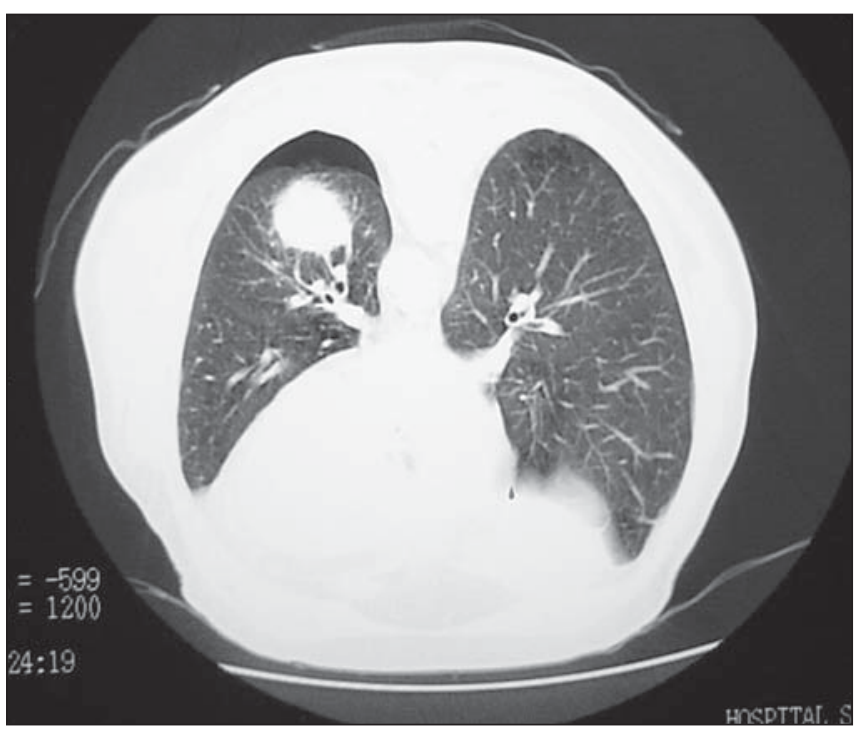

B

Figura 4. Paciente de 75 anos de idade submetido a laringectomia devido a carcinoma espinocelular de laringe, que evolui com massa no lobo inferior do pulmão esquerdo, cujo diagnóstico pela biópsia por agulha fina confirmou carcinoma indiferenciado de grandes células. A: Imagem obtida para confirmação da posição da agulha de biópsia no interior da lesão. B: Imagem de controle após a realização da biópsia, que evidencia pequeno pneumotórax.

para lesões com diâmetro médio de $2,9 \mathrm{~cm}$, Haramati e Austin ${ }^{(\mathbf{1 6})}$ relataram $81 \%$ para lesões de 4,0 cm e Lucidarme et al. ${ }^{(\mathbf{8})}$ relataram $88 \%$ para lesões com $3,36 \mathrm{~cm}$ em média.

Outro dado importante citado em estudos anteriores é que a acurácia aumenta se aumentarmos o número de passagens da agulha através da lesão. Na técnica convencional isso implicaria maior número de transgressões da pleura, o que aumentaria o risco de complicações. Com a técnica coaxial, o número de transgressões pleurais limita-se a uma, pois após a passagem da agulha-guia (externa), retira-se a quantidade necessária de material por meio dela, sem deslocá-la, utilizando uma agulha mais fina que é passada pelo interior da agulhaguia. Não se utilizou essa técnica no presente estudo, pela não disponibilidade do material apropriado, que foi adquirido posteriormente.

Vale salientar que em centros onde se dispõem de TC em tempo real (TC fluoroscopia) a acurácia pode ser ainda maior. Katada et al. ${ }^{(17)}$ relataram acurácia de $100 \%$ para biópsias aspirativas por agulhas finas, guiadas por TC fluoroscopia para lesões maiores que $1,1 \mathrm{~cm}$ e $67 \%$ para lesões menores que $1,0 \mathrm{~cm}$. $\mathrm{O}$ grande pro- 


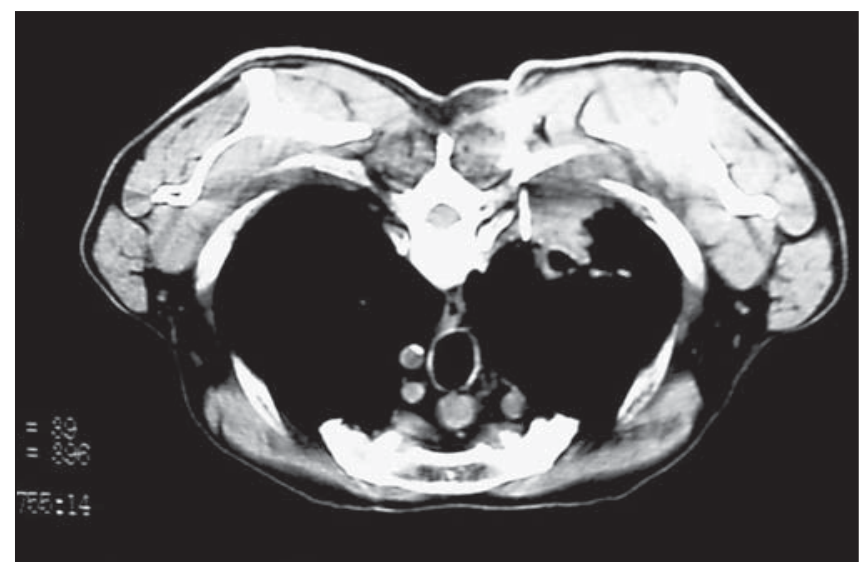

A

Paciente de 60 anos de idade, tabagista de

Figura 5. Paciente de 60 anos de idade, tabagista de longa data, com lesões metastáticas cerebrais e no lobo superior do pulmão direito, foi submetida a biópsia aspirativa por agulha fina e biópsia por fragmentos, que evidenciaram carcinoma indiferenciado de grandes células. Logo após a biópsia o paciente evoluiu com escarros com raias de sangue. A: Agulha de biópsia localizada no interior da lesão. B: Imagem obtida após o procedimento, que evidencia hemorragia perilesional.

blema é a exposição das mãos do profissional à radiação e a não disponibilidade do aparelho de TC em tempo real na maioria dos centros.

Outra técnica que vem sendo utilizada na tentativa de melhorar a acurácia é a congelação do fragmento obtido pela biópsia, com pronta avaliação pelo patologista ${ }^{(11)} \mathrm{e}$ a utilização da técnica de controle da respiração "respiratory gating". Todas essas técnicas oneram os custos do procedimento, inviabilizando a sua realização na grande maioria das instituições do país, além de necessitarem de maiores estudos para confirmação do seu real valor.

A escolha do método a ser utilizado para realização da biópsia ainda é motivo de discussão na literatura, não existindo consenso quanto à escolha do melhor método para cada situação em relação ao tamanho da lesão e à distância da pleura à lesão, entre outras. A biópsia aspirativa por agulha fina apresenta, como maior desvantagem, a menor acurácia no diagnóstico específico de lesões que não são neoplásicas $^{(4)}$ e a necessidade de um citopatologista na sala durante o procedimento para análise das amostras, fato indispensável para reduzir o número de biópsias inconclusivas $^{(5,12)}$. A grande vantagem teórica da biópsia aspirativa por agulha fina sobre a biópsia por fragmentos seria a menor incidência de complicações, fato controverso na literatura ${ }^{(\mathbf{1 - 1 2})}$.

As complicações mais freqüentes são o pneumotórax e a hemorragia pulmonar perilesional. Os principais fatores relacionados ao aumento do risco de pneumotórax são o tamanho da lesão (lesões pequenas apresentam um maior risco), a presença de enfisema e o contato da lesão com a pleura (quanto maior o contato menor o risco de pneumotórax $)^{(7)}$.

Quando o pneumotórax ocorre no momento da passagem da agulha pela pleura, ocorre colapso parcial do pulmão, mudando a posição inicial da lesão com relação à marcação cutânea, dificultando o acesso à lesão, principalmente no caso de lesões pequenas. Neste estudo, essa situação ocorreu três vezes, sendo que, em uma delas, devido ao diâmetro da lesão $(5,0 \mathrm{~cm})$, não houve problemas.

Nenhum caso de pneumotórax do presente estudo necessitou de dreno torácico. Apenas dois foram submetidos à aspiração com gelco 14 devido às suas maiores dimensões.

A hemorragia perilesional ocorreu em dez pacientes, sem relatos de hemoptise significativa ou outras complicações citadas em outros estudos ${ }^{(\mathbf{4 , 1 1}, 17)}$.

Como os nódulos sofrem alteração da sua posição com a respiração, a colaboração do paciente foi fundamental e indispensável para o sucesso do procedimento, a orientação e o treinamento do paciente antes da realização do exame. Além disso, o procedimento e possíveis complicações devem ser explicados aos pacientes, fato que geralmente os tranquiliza, facilitando a realização do exame.

\section{CONCLUSÃO}

A acurácia da biópsia aspirativa e por fragmento de lesões pulmonares depende do tamanho da lesão e da colaboração do paciente. Quando realizados por profissional experiente, esses procedimentos mostraram-se relativamente seguros e com índices satisfatórios de acurácia.

\section{REFERÊNCIAS}

1. vanSonnenberg E, Casola G, Ho M, et al. Difficult thoracic lesions: CT-guided biopsy experience in 150 cases. Radiology 1988;167:457-461.

2. Westcott JL, Rao N, Colley DP. Transthoracic needle biopsy of small pulmonary nodules. Radiology 1997;202:97-103.

3. Westcott JL. Direct percutaneous needle aspiration of localized pulmonary lesions: results in 422 patients. Radiology 1980;137:31-35.

4. Yu LS, Deheinzelin D, Younes RN, Chojniak R. Computed tomography-guided cutting needle biopsy of pulmonary lesions. Rev Hosp Clin Fac Med S Paulo 2002;57:15-18.

5. Wallace MJ, Krishnamurthy S, Broemeling LD, et al. CT-guided percutaneous fine-needle aspiration biopsy of small $(<$ or $=1-\mathrm{cm})$ pulmonary lesions. Radiology 2002;225:823-828.

6. Brown KT, Brody LA, Getrajdman GI, Napp TE. Outpatient treatment of iatrogenic pneumothorax after needle biopsy. Radiology 1997;205:249 252.

7. Cox JE, Chiles C, McManus CM, Aquino SL, Choplin RH. Transthoracic needle aspiration biopsy: variables that affect risk of pneumothorax. Radiology 1999;212:165-168.

8. Lucidarme O, Howarth N, Finet JF, Grenier PA. Intrapulmonary lesions: percutaneous automated biopsy with a detachable, 18-gauge, coaxial cutting needle. Radiology 1998;207:759-765.

9. Tomiyama N, Mihara N, Maeda M, et al. CTguided needle biopsy of small pulmonary nodules: value of respiratory gating. Radiology 2000; 217:907-910. 
10. Tsukada H, Satou T, Iwashima A, Souma T. Di agnostic accuracy of CT-guided automated needle biopsy of lung nodules. AJR Am J Roentgenol 2000;175:239-243.

11. Hayashi N, Sakai T, Kitagawa M, et al. CT-guided biopsy of pulmonary nodules less than $3 \mathrm{~cm}$ : usefulness of the spring-operated core biopsy needle and frozen-section pathologic diagnosis. AJR Am J Roentgenol 1998;170:329-331.

12. Li H, Boiselle PM, Shepard JO, Trotman-Dickenson B, McLoud TC. Diagnostic accuracy and safety of CT-guided percutaneous needle aspiration biopsy of the lung: comparison of small and large pulmonary nodules. AJR Am J Roentgenol 1996;167:105-109.

13. Santos GC, Morini SR, Granero LC, Chojniak R, Longatto Filho A. Fine needle aspiration of thoracic lesions: experience in a Brazilian cancer center. Pathologica 1999;91:256-259.

14. Junqueira MAF, Filho MT, Vargas FS, et al. Biópsia pulmonar transtorácica orientada por tomografia computadorizada. J Pneumol 1990;16:1-5.
15. Klein JS, Salomon G, Stewart EA. Transthoracic needle biopsy with a coaxially placed 20 -gauge automated cutting needle: results in 122 patients. Radiology 1996;198:715-720.

16. Haramati LB, Austin JHM. Complications after CT-guided needle biopsy through aerated versus nonaereted lung. Radiology 1991;181:778.

17. Katada K, Kato R, Anno H, et al. Guidance with real-time CT fluoroscopy: early clinical experience. Radiology 1996;200:851-856. 